\title{
Comprometimento e consentimento organizacional: um estudo da validade discriminante dos construtos
}

\author{
Adriano de Lemos Alves Peixoto - Universidade Federal da Babia, Salvador, Brasil \\ Antônio Virgílio Bittencourt Bastos - Universidade Federal da Babia, Salvador, Brasil \\ Icléia Santos Dorea Soares - Universidade Federal da Babia, Salvador, Brasil \\ Júlia Rodrigues Lobo - Universidade Federal da Babia, Salvador, Brasil
}

\begin{abstract}
Resumo
O comprometimento organizacional é um dos tópicos de pesquisa mais investigados no campo do comportamento organizacional e a gênese da sua importância reside no reconhecimento do amplo poder explicativo do construto. Tendo em vista a aparente sobreposição reportada na literatura entre a dimensão afetiva e a dimensão normativa do comprometimento, foi proposto o construto de consentimento para diferenciar trabalhadores comprometidos (vínculo ativo) de obedientes (vínculo passivo). O presente artigo busca contribuir para estabelecer os limites entre os construtos do comprometimento e consentimento organizacionais. O estudo consistiu em um survey de corte transversal entre 994 trabalhadores de diferentes organizações e segmentos produtivos. A análise sugere indícios de validade discriminante entre os construtos como se pode perceber, por exemplo, em relação às diferenças na magnitude do efeito das variáveis sociodemográficas na explicação das duas bases do comprometimento e do consentimento e pela baixa correlação entre o consentimento a base normativa $(r=0,240)$.

Palavras-chave: comprometimento organizacional; vínculos; validade; obediência.
\end{abstract}

\section{Organizational commitment and compliance: a study on the discriminant validity between constructs}

\begin{abstract}
The organizational commitment is one of the most investigated topics of research in the field of organizational behavior and the genesis of its importance lies in recognizing the broad explanatory power of the construct. Given the apparent overlap reported in the literature between the affective and the normative dimensions of commitment, it has been proposed the construct of organizational compliance to differentiate committed workers from obedient ones. This article aims to establish the boundaries between the constructs of organizational commitment and consent. The study consisted of a cross-sectional survey with 994 workers from different organizations and production segments. The analysis suggests evidence of discriminant validity between consent, affective commitment and normative commitment. This can be observed in the different effect size of socio demographic variables on commitment and consent and the low correlation between consent and normative base of commitment $(r=240)$.

Keywords: organizational commitment; compliance; validity; attachment behaviour.
\end{abstract}

\section{Compromiso y consentimiento organizacional: un estudio de validez discriminante de los constructos}

\section{Resumen}

El compromiso organizacional es uno de los temas más investigados en el campo del comportamiento organizacional y su importancia radica en el reconocimiento del amplio poder explicativo del constructo. Dada la aparente superposición entre la dimensión afectiva y la dimensión normativa del compromiso registrada en la literatura, fue propuesto el constructo del consentimiento para diferenciar los trabajadores comprometidos (vínculo activo) de los obedientes (vínculo pasivo). Este artículo busca contribuir para establecer los límites entre los constructos del compromiso y el consentimiento organizacional. El estudio consistió en un survey transversal con 994 trabajadores de diferentes organizaciones y segmentos productivos. El análisis señala indicios de validez discriminante entre los constructos, como puede verse por ejemplo, en relación a las diferencias en la magnitud del efecto de las variables socio-demográficas, en la explicación de las dos bases del compromiso y el consentimiento y por la baja correlación entre la base normativa y el consentimiento $(r=0,240)$.

Palabra-clave: compromiso organizacional; vínculos; validez; obediencia.

\section{Introdução}

O comprometimento organizacional (CO) é um dos tópicos de pesquisa mais investigados no campo do comportamento organizacional. Seu estudo data desde o final da década de 1960 (Silva, 2009), sendo que a quantidade e a frequência de pesquisas ao longo desse meio século refletem o reconhecimento do conceito como explicativo de uma série de comportamentos relevantes para a organização (Bastos, Rodrigues, Macambira, \& Borges-Andrade, 2014) como, por exemplo, desempenho e satisfação no trabalho (Imran, Arif, Cheema, \& Azeem, 2014).

Tendo em vista a sua importância, um grande esforço tem sido despendido na tentativa de melhor estabelecimento de seus limites conceituais (por 
exemplo: Becker, 1960; Ko, Price, \& Mueller, 1997; Meyer \& Allen, 1997; Solinger, Olffen, \& Roe, 2008) uma vez que o comprometimento é um conceito polissêmico. Nesse sentido, duas abordagens distintas disputam entre si o espaço de melhor modelo explicativo. Se de um lado existe uma corrente teórica que busca consolidar os vários sentidos possíveis para o construto, caracterizando a abordagem tridimensional do comprometimento (ex.: Meyer \& Allen, 1997), do outro lado, encontram-se aqueles pesquisadores que favorecem uma abordagem mais direta e que propõem um construto livre de tensões e contradições, tanto conceituais quanto metodológicas (ex.: Sollinger et al., 2008), focando sua atenção no que seria a dimensão central do comprometimento, caracterizando, assim, um modelo unidimensional (Mowday, Steers, \& Porter, 1979).

Vários foram os autores que, ao longo do tempo, propuseram e defenderam uma natureza multidimensional para o comprometimento, considerando os diferentes processos psicossociais que podem estar na base desse vínculo. Entre eles, destacam-se as proposições de O’Reilly e Chatman (1986) e Jaros (1997), sendo o modelo tridimensional sistematizado por Meyer e Allen $(1991,1997)$ a teoria de maior poder explicativo e a mais aceita atualmente. De um modo geral, esses autores afirmam ser o comprometimento um construto multidimensional composto pelas bases afetiva, normativa e de continuação (algumas vezes também chamada de base instrumental) (Bastos, Rodrigues, Moscon, Silva, \& Pinho, 2013). Essas dimensões podem ser resumidamente definidas da seguinte forma: a base afetiva (CA) é caracterizada pelo desejo do sujeito em permanecer na organização em virtude de seu envolvimento e identificação com valores e propósitos organizacionais (Mowday, Porter, \& Steers, 1982); a base normativa $(\mathrm{CN})$ é definida por uma obrigação moral sentida pelo indivíduo em permanecer na organização, por acreditar ter o dever de retribuir os investimentos recebidos, em termos de treinamentos e desenvolvimento pessoal. Por fim, a base de continuação ou instrumental (CI) se caracteriza pela percepção do indivíduo de que os custos associados a sua saída da organização são muito elevados, seja em função da perda de sua rede de relacionamentos, seja em função da perda de benefícios adquiridos (Meyer \& Allen, 1997).

Várias críticas foram sendo feitas, especialmente na última década, ao modelo tridimensional de Meyer e Allen (1991), tanto no tocante aos limites conceituais entre bases do comprometimento, quanto às inconsistências empíricas entre preditores e consequentes das diferentes bases (Rodrigues \& Bastos, 2012; Rodrigues Bastos \& Gondim, 2013). Diversos autores (Ko et al.,1997; Bergman, 2006; Solinger et al., 2008) apontam as similaridades existentes entre os preditores das bases normativa e afetiva do comprometimento, sugerindo uma possível sobreposição entre ambas. De forma contrária, são muito distintos os preditores e os consequentes das bases afetiva e de continuação, sugerindo tratarem-se de dois construtos distintos (Bastos, 1994; Carvalho, Alves, Peixoto, \& Bastos, 2011; Rodrigues \& Bastos, 2009). Adicionalmente, as escalas propostas no modelo, sobretudo aquela que mensura o comprometimento de continuação, não apresentam bons indicadores psicométricos em culturas distintas da estadunidense. Em função dessas dificuldades, alguns autores defendem a retomada do modelo unidimensional (Solinger et al., 1998; Silva, 2009).

Inserido nessa perspectiva de redução da extensão assumida pelo conceito de comprometimento no modelo tridimensional proposto por Meyer e Allen (1991, 1993), foi apresentado por Silva (2009) e Silva e Bastos (2010) o construto denominado consentimento organizacional, para abarcar o conjunto de ideias associadas às bases de compliance (O'Reilly \& Chatman, 1986) e da própria base normativa do modelo tridimensional. A noção de uma adesão, aquiescência ou mesmo obediência àquilo que a organização espera do trabalhador é tomada, nessa proposta, como um tipo específico de vínculo, de natureza passiva, que se diferenciaria do comprometimento, agora entendido como um vínculo ativo, de natureza afetiva e fundada em uma efetiva identificação entre valores pessoais e organizacionais e que conduz a desempenhos e contribuições para além do papel prescrito pela organização.

Um dos principais problemas apontados no modelo de Meyer e Allen $(1991,1997)$ consiste no agrupamento em um construto de bases com diferentes alvos/focos, conforme afirmam Solinger et al. (2008). A dimensão afetiva teria como foco um objeto, a organização, enquanto as dimensões de continuação e normativa teriam como foco um comportamento - o de permanecer ou não trabalhando na organização. Os autores também sugerem que ele não deveria ser usado como modelo geral de comprometimento organizacional, mas sim como modelo específico de predição de turnover, já que a caracterização das dimensões propostas é centrada na capacidade das bases do comprometimento de predizerem a permanência do indivíduo na organização. Essa crítica já 
fora feita por Jaros (1997) ao sugerir que as intenções de turnover seriam a ligação conceitual entre as diversas bases do comprometimento formuladas por Meyer e Allen (1991).

Uma segunda linha de crítica ao modelo tridimensional pode ser encontrada em Ko et al. (1997). Para os autores Meyer e Allen (1990, 1991), não oferecem uma definição precisa de comprometimento que inclua aspectos afetivos, normativos e de continuação, definindo o construto apenas como um vago "estado psicológico" - sem explicitar o que exatamente se entenderia por esse estado. $O$ fato de o modelo proposto ser referido pelos próprios autores como uma "expansão do construto" (Meyer \& Allen, 1991), oriundo da tentativa de achar uma ligação entre diversas conceitualizações de comprometimento, pode ter contribuído para que o conceito tenha se tornado vago.

Uma terceira linha das críticas refere-se às dificuldades na delimitação interdimensional que têm sido apontadas, principalmente, em relação às bases normativa e afetiva, que apresentam altos índices de correlação entre si (Jaros, 1997; Ko et al., 1997; Solinger et al.,, 2008; Bandeira, Marques \& Veiga, 2000; Blau, 2001; Meyer \& Herscovitch, 2001; Meyer, Stanley, Herscovitch \& Topolnytsky, 2002). Ko, Price e Mueller (1997), por exemplo, encontraram correlações positivas entre as dimensões afetiva e normativa maiores que $r=0,70$. Nessa mesma direção, percebe-se que a maioria das variáveis antecedentes da base normativa também aparece como preditora da base afetiva. Nesse modelo de três bases, a dimensão que apresenta os maiores índices de correlação positiva com comportamentos relevantes para a organização, tais como esforço discricionário e desempenho, é a dimensão afetiva. Da mesma forma, ela demonstra ter uma correlação negativa com o absenteísmo e turnover mais significativa que as outras duas (Meyer \& Allen, 1997), o que explica a predominância de estudos que enfatizam os preditores e consequentes do comprometimento afetivo. Por outro lado, a base normativa, também, apresenta fortes correlações positivas com os comportamentos desejáveis pela organização, porém não alcançando os mesmos valores indicados pela dimensão afetiva. Tais achados levaram os autores a concluir que a base normativa do comprometimento teria pouca validade discriminante em relação à base afetiva e, por esse motivo, o comprometimento normativo poderia ser considerado como um conceito redundante.
Evidências empíricas dessa "aproximação" entre as bases afetivas e normativas são abundantes. Por exemplo, no estudo feito por Gellatly, Meyer e Luchak (2006) são apresentadas correlações entre essas duas bases do comprometimento com a variável intenção de permanecer na organização $(r=0,46$, para $\mathrm{CN} ; r=0,43$, para CA). Entretanto, é preciso reconhecer que existem poucas pesquisas que enfatizam a discriminação entre os antecedentes, consequentes e correlatos do comprometimento, focalizando, especificamente, essa relação entre as bases normativa e afetiva, o que tornaria possível a diferenciação mais clara entre ambos (Bergman, 2006).

Bandeira, Marques e Veiga (2000) também encontraram grande similaridade entre os antecedentes das duas dimensões em questão. Os autores obtiveram correlações de $r=0,50$ e $r=0,51$ entre políticas de treinamento e desenvolvimento, e comprometimento normativo e afetivo, respectivamente. Também foram encontradas correlações bastante próximas entre o relacionamento do empregado com a chefia com a base normativa $(r=0,47)$ e a base afetiva $(r=0,44)$. Além disso, a meta-análise realizada por Meyer, Stanley, Herscovitch e Topolnystsky (2002) não identificou antecedentes específicos para o comprometimento normativo e encontrou correlação de $r=0,63$ entre o comprometimento normativo e o afetivo, o que demonstra a dificuldade de diferenciação entre as bases afetiva e normativa reiteradamente discutida na literatura.

Outro importante foco de críticas tem sido direcionado à qualidade das escalas produzidas, suas inconsistências empíricas e a validade discriminante entre as bases (Bastos, 1994; Medeiros, 2003; Costa, 2005; Solinger et al., 2008). Medeiros (2003) afirma que, nas validações da escala feitas no Brasil, foram encontrados índices apenas moderados de consistência interna entre as dimensões do comprometimento, além de baixo percentual da variação total explicada.

Para Bergman (2006) essa aparente sobreposição entre as bases normativa e afetiva decorre de mensurações que não correspondem corretamente à teoria. Para a autora, alguns fatores podem estar na origem da confusão conceitual e empírica dos construtos. Uma das problemáticas pode estar relacionada à utilização de termos referentes a sentimentos, tais como "sinto que" nas escalas de comprometimento normativo. Esse uso pode fazer com que o respondente encare os itens com um teor mais sentimental do que o desejado. Essas sutilezas devem ser levadas em consideração na construção das escalas, de modo que não haja confusão entre o 
desejo de participar da organização e a obrigação em nela permanecer.

Todavia, Jaros (1997) argumenta que a utilização de termos relacionados a sentimentos nas escalas é proveniente da própria definição dada por Meyer \& Allen (1997) aos construtos. O modo como o comprometimento normativo é conceituado - como um "sentimento" de obrigação moral - supõe um vínculo baseado em sentimentos, afetos, o que pode acarretar na sobreposição com o comprometimento afetivo.

Referindo-se à influência de outra variável na relação entre o comprometimento afetivo e o normativo, Bergman (2006) aponta a interferência da cultura no modo como uma obrigação é sentida e expressa. A autora argumenta que o motivo de surgimento de uma obrigação, assim como suas normas específicas, pode variar dependendo da cultura. Janoff-Bulman e Leggatt (2002) parecem corroborar essa hipótese quando afirmam que culturas individualistas costumam valorizar, principalmente, a liberdade individual, enquanto culturas coletivistas costumam enfatizar o bem-estar coletivo e papéis sociais em detrimento de objetivos pessoais. Logo, em culturas coletivistas, as obrigações sociais para com grupos são reconhecidas não só como um dever, mas também como um querer. Para os indivíduos desse tipo de cultura a "obrigação moral" presente no comprometimento normativo pode ser também um desejo, facilmente confundível com o comprometimento afetivo.

Visto as inconsistências empíricas e os problemas conceituais mais profundos acima descritos e apontados por autores como Bastos (1994), Ko, Price e Mueller (1997), e Solinger, Olffen e Roe (1998), várias são as direções apontadas pela literatura na tentativa de melhor delimitar, conceitual e empiricamente, o fenômeno do comprometimento no trabalho (Cooper-Hakim \& Viswesvaran, 2005; Rodrigues, 2009; Silva, 2009).

Dentre essas, destacam-se as de Rodrigues (2009) e Silva (2009) de resgatar a visão do comprometimento organizacional como um construto unidimensional, como originariamente postulado por Mowday, Porter e Steers (1982), uma vez que a validade de construto das dimensões normativa e de continuação não estaria tão bem consolidada quanto a da base afetiva.

Como proposta de resolução dessa superposição entre as dimensões, e numa tentativa de solucionar os problemas citados na literatura que se referem a aspectos metodológicos relacionados à mensuração do construto, Silva (2009) e Silva e Bastos (2010) propõem um novo vínculo diferenciado do comprometimento e baseado na noção de obediência. Para os autores, esse novo construto seria o que eles chamam de consentimento organizacional.

Nessa direção, eles propõem o resgate da ideia de compliance apresentada originalmente por O'Reilly e Chatman (1986) que, em conjunto com a literatura sociológica (Halaby, 1986; Weber, 1994 citado por Silva, 2009), oferecem base para a definição de um tipo de vínculo do indivíduo pautado pela internalização do papel de subordinação presente nas relações de trabalho. Esse papel seria capaz de determinar a relação desse trabalhador com as normas e regras da organização. Trata-se de um tipo de vínculo que se aproxima do que Bar-Hayim e Berman (1992) identificaram como sendo um comprometimento passivo, associado a comportamentos de lealdade à organização.

O consentimento, de acordo com Silva (2009), seria definido como a tendência do indivíduo em obedecer ao seu superior hierárquico no ambiente de trabalho, representando um vínculo estabelecido pelo indivíduo com a organização. Com base na Psicologia Social e na Sociologia, ela aponta um modelo para o consentimento organizacional com uma estrutura bifatorial. O primeiro fator, denominado obediência cega, representaria um cumprimento "automático" da ordem de um superior, sem uma avaliação ou julgamento a seu respeito pelo subordinado, ou ainda, o cumprimento de uma ordem mesmo quando o indivíduo apresenta discordância em relação ao seu cumprimento. Já o segundo fator, que foi por ela denominado aceitação íntima, se caracterizaria pela realização de tarefas, marcada pela concordância com as regras e normas estabelecidas pela organização, acarretando em identificação e internalização das normas da organização.

Ao reconhecer que a proposta dada por Silva (2009) busca solucionar algumas das dificuldades aqui elencadas e apontadas pela literatura do comprometimento, em especial àquelas relacionadas à superposição das bases afetiva e normativa do comprometimento, na seção seguinte, testa-se o modelo proposto, buscando identificar se alguns dos problemas apontados, de fato, são superados. Para tal, será investigada a validade discriminante entre o consentimento, comprometimento afetivo e o normativo por meio da análise de validade discriminante entre os construtos com os antecedentes do comprometimento descritos na literatura.

O presente artigo, que busca contribuir para estabelecer os limites ente os construtos de comprometimento 
e consentimento organizacionais, encontra-se dividido em quatro seções além dessa introdução. $\mathrm{Na}$ seção seguinte, será apresentada a metodologia utilizada na investigação do problema, seguida de uma seção com a apresentação dos resultados e discussão, e uma seção com as principais conclusões deste trabalho.

\section{Método}

\section{Participantes}

A coleta de dados foi realizada após aprovação do projeto pelo Comitê de Ética em Pesquisa da Faculdade de Filosofia e Ciências Humanas da UFBA. Os dados analisados neste artigo foram coletados com base em questionários individualmente aplicados em uma amostra não probabilística composta por 994 trabalhadores técnicos, administrativos e operacionais, de diversas empresas públicas e privadas, de ambos os sexos $(59 \%$ do sexo feminino), em várias ocupações e distintos segmentos de atuação. A escolaridade dos trabalhadores foi bastante variada, cerca de 38,4\% tinha, no máximo, ensino médio completo; $35,2 \%$ ingressou mas não concluiu o ensino superior, e 10,8\% finalizou a graduação. A maior parte dos sujeitos tinha entre 20 e 37 anos de idade $(78,5 \%)$ e trabalhava em empresas privadas $(83,5 \%)$ com mais de 100 funcionários $(77,2 \%)$. Cerca de metade dos participantes (48,1\%) estava há dois anos ou mais na empresa em que trabalhava quando a pesquisa foi realizada.

\section{Instrumento}

Utilizou-se um questionário contendo 23 itens para mensurar as variáveis dependentes do estudo - comprometimento afetivo, comprometimento normativo e consentimento. Tais itens estão distribuídos da seguinte forma: nove itens correspondentes à escala de comprometimento organizacional afetivo (CA) construída e validada por Bastos (1994); nove itens compondo a escala consentimento (dois itens correspondendo à dimensão aceitação íntima e sete itens à dimensão obediência cega) extraída de Silva (2009); e cinco itens referentes à escala de comprometimento organizacional normativo (CN) (Bastos, 1994). Os itens foram respondidos por meio de escalas do tipo Likert de seis pontos ( $1=$ "discordo totalmente" a $6=$ "concordo totalmente").

Mowday, Steers e Porter (1979) e Mathieu e Zajac (1990) asseguram que seria útil aprender mais sobre os principais antecedentes e consequentes do comprometimento organizacional $(\mathrm{CO})$, tendo em vista um reconhecimento maior do poder de alcance e limites do construto. Essa preocupação em identificar as fronteiras do $\mathrm{CO}$ é proveniente dos diversos preditores identificados e apontados pela literatura da área (Blau, 2001; Klein, Becker \& Meyer, 2010; Meyeret al., 2002). Diante de tal quadro, o presente estudo optou por acrescentar, em seu instrumento, um conjunto de variáveis independentes desenvolvidas para esta pesquisa e que expressam políticas e práticas na área de gestão de recursos humanos que, se adequadamente aplicadas, seriam capazes de favorecer o desenvolvimento do comprometimento nos trabalhadores. São elas: treinamento e desenvolvimento, com cinco itens $(\alpha=0.774)$; remuneração, com três itens $(\alpha=0.733)$; avaliação do chefe, com seis itens $(\alpha=0.923$.), coordenação e controle, com quatro itens $(\alpha=0.625)$; avaliação da equipe de trabalho com três itens $(\alpha=0.723)$.

O instrumento foi, ainda, composto por um conjunto de variáveis sociodemográficas que expressam características gerais das organizações e dos empregados, tais como: tamanho da empresa, tempo de empresa, ano de nascimento e escolaridade. Essas variáveis, também, têm sido apontadas na literatura como possíveis antecedentes do comprometimento (Meyer \& Allen, 1997; Wright \& Kehoe, 2009).

\section{Procedimentos de análise}

Foram realizadas análises correlacionais e de regressão múltipla entre $\mathrm{CA}, \mathrm{CN}$ e o consentimento e destes com as variáveis sociodemográficas e de políticas de gestão, para avaliar a relação entre os construtos e estabelecer sua validade discriminante.

\section{Resultados e Discussão}

Um pressuposto básico para a aceitação da proposta do consentimento como um vínculo distinto do comprometimento consiste na superação de alguns dos problemas metodológicos e conceituais já identificados e associados à relação entre o comprometimento normativo e o afetivo. Isso posto a avaliação do modelo sugerido por Silva (2009), se desenvolverá em etapas distintas. Inicialmente, busca-se identificar a relação existente entre as bases afetiva e normativa do comprometimento e o consentimento. Em seguida, será feita uma análise de regressão entre as duas dimensões do comprometimento e o consentimento em função de um conjunto de práticas e políticas de gestões usualmente consideradas antecedentes do comprometimento organizacional. Esse mesmo procedimento se repetirá em 
relação a um conjunto de variáveis sociodemográficas. A análise se inicia buscando observar a correlação entre os diversos construtos, conforme pode ser percebido na tabela a seguir.

Os resultados encontrados apontam para uma correlação positiva entre o $\mathrm{CA}$ e o $\mathrm{CN}(\mathrm{r}=0,399$, $\mathrm{p}<001)$ em linha com os achados relatados anteriormente na literatura como spode ser visto, por exemplo, em Ko, Price e Mueller (1997), Bergman (2006) e Solinger et al. (2008), dando suporte à noção de alguma superposição conceitual entre as bases do comprometimento, ainda que em grau menor do que o apontado na literatura.

Já os resultados referentes à correlação entre $\mathrm{o}$ consentimento e as bases afetiva $(r=0,218, p<001)$ e normativa $(\mathrm{r}=0,240, \mathrm{p}<001)$ do comprometimento podem ser considerados indícios da validade discriminante entre os construtos. De qualquer forma, considerando que todos os três construtos se referem a vínculos que os trabalhadores estabelecem com as organizações, algum grau de correlação entre eles é esperado. Essses achados iniciais sugerem que o consentimento, na medida proposta por Silva (2009), afasta-se do CA e do $\mathrm{CN}$ como previsto pelo modelo. $\mathrm{Na}$ tabela abaixo, observa-se os resultados das análises de regressão do $\mathrm{CA}, \mathrm{CN}$ e do consentimento em relação a um conjunto de variáveis sociodemográficas.

As diferenças na magnitude do efeito das variáveis sociodemográficas na explicação das duas bases do comprometimento e do consentimento reforçam a noção de um distanciamento entre os tipos de vínculos. Percebe-se que existe um padrão muito distinto de relação entre os preditores (antecedentes) investigados e os diversos vínculos, chamando particular atenção o tamanho do efeito da escolaridade. Além disso, a escolaridade explica importante variação do consentimento $(29,2 \%)$, ao passo que seu poder explicativo em relação à variação observada no CA e no $\mathrm{CN}$ é muito baixa, girando em torno de $6 \%$, em ambos os casos.

Como descrito anteriormente, o consentimento (Silva, 2009) se assemelha conceitualmente a um vínculo passivo, associado à lealdade, como proposto por Bar-Hayim e Berman (1992). Em seu estudo, a escolaridade também se relaciona, negativamente, com o vínculo por eles apresentado. Os autores atribuem esse resultado às limitações no mercado de trabalho que sujeitos de menor escolaridade teriam, fazendo

Tabela 1. Coeficientes de Correlação entre o Comprometimento Afetivo, Normativo e o Consentimento

\begin{tabular}{lccc}
\hline & $\begin{array}{c}\text { Comprometimento } \\
\text { afetivo }(\mathrm{CA})\end{array}$ & $\begin{array}{c}\text { Comprometimento } \\
\text { normativo }(\mathrm{CN})\end{array}$ & Consentimento \\
\hline Comprometimento afetivo $(\mathrm{CA})$ & - & - & \\
Comprometimento normativo $(\mathrm{CN})$ & $0,399^{* *}$ & $0,240^{* *}$ & ---- \\
Consentimento & $0,218^{* *}$ & - \\
\hline
\end{tabular}

$\mathrm{N}=994, * * \mathrm{p}<.001$.

Tabela 2. Efeitos da regressão de Comprometimento Afetivo, Normativo e Consentimento com Variáveis Sociodemográficas

\begin{tabular}{lccc}
\hline & Comprometimento afetivo & Comprometimento normativo & Consentimento \\
\hline Escolaridade & $-0,183^{* *}$ & $-0,253^{* *}$ & $-0,545^{* *}$ \\
Idade & $-0,158^{* *}$ & $-0,002$ & 0,060 \\
Tempo na empresa & 0,036 & $-0,003$ & 0,012 \\
Tamanho da empresa & 0,053 & $-0,020$ & 0,007 \\
$\mathrm{R}$ & 0,259 & 0,254 & 0,543 \\
$\mathrm{R}^{2}$ & 0,063 & 0,061 & 0,292 \\
\hline
\end{tabular}

** $\mathrm{p}<0,001$ 
com que eles se vinculassem mais fortemente à organização em que trabalham, porém de forma passiva. Essa explicação pode ser atribuída também ao grau e direção da influência da escolaridade em relação ao consentimento. Adicionalmente, pode-se propor que pessoas com menor grau de escolaridade tendem a aceitar e a obedecer às ordens e normas da organização de uma forma mais acrítica, justificando os resultados encontrados.

Em suma, o efeito diferenciado das variáveis socioambientais nos vínculos do indivíduo com a organização, especialmente em relação ao consentimento, sugerem construtos distintos indicando haver validade discriminante entre eles. Na sequência, é analisado o impacto das variáveis denominadas políticas e práticas de gestão nas bases do comprometimento e no consentimento cujos resultados encontram-se na Tabela 3.

O quadro que emerge da análise do impacto das políticas e práticas de gestão nas variáveis dependentes segue, aproximadamente, o mesmo padrão daquele observado em relação às variáveis demográficas. É possível perceber uma aproximação entre os resultados encontrados (tamanho dos efeitos) para o $\mathrm{CN}$ e o CA em linha com as previsões da literatura, como pode ser notado pelo efeito do treinamento e desenvolvimento e da remuneração nos vínculos. Simultaneamente, há um afastamento entre os construtos na medida em que a avaliação da equipe e do chefe é significativa na explicação do CA, mas não contribuem para a explicação do CN. Em outras palavras, fica claro que diferentes práticas e políticas de gestão impactam de forma distinta esses dois construtos, o que aponta para uma validade discriminante entre eles, ao passo que as aproximações podem ser explicadas a partir da superposição conceitual já apontada e amplamente discutida na literatura.

Por outro lado, o impacto das práticas de gestão investigadas é muito mais importante e significativo na previsão do CA (38,4\% da variação explicada) quando comparado ao $\mathrm{CN}$ (16,5\% da variação explicada). Mais especificamente, o efeito do treinamento e desenvolvimento no CA pode ser explicado pelo que foi exposto por Meyer e Allen (1997). Os autores afirmam que a noção que um funcionário possui das próprias competências é um importante fator no desenvolvimento do comprometimento organizacional e, como treinamento e desenvolvimento (T\&D) permitem o aprimoramento das competências pessoais e profissionais, a percepção individual sobre elas torna-se, então, mais refinada. Desse modo, é de se esperar que o efeito de T\&D para a explicação do comprometimento afetivo seja significativa $(\beta=0,255)$.

Quanto ao CN, o impacto da mesma variável pode ser explicado por meio do exemplo do impacto dos treinamentos na socialização dos funcionários. Segundo Meyer e Allen (1997), a socialização é um elemento que deve ser considerado na explicação do comprometimento normativo, pois nela é feita a transmissão dos valores organizacionais e dos comportamentos esperados, como, por exemplo, lealdade para com a organização. Do mesmo modo que o T\&D permite o desenvolvimento das competências dos funcionários e, assim, acaba por anteceder o surgimento do CA, a atividade de treinamento e desenvolvimento também permite o aparecimento do $\mathrm{CN}$, pois o funcionário

Tabela 3. Efeitos da Regressão entre Comprometimento Afetivo, Normativo e Consentimento com Políticas e Práticas de Gestão

\begin{tabular}{lccc}
\hline & $\begin{array}{c}\text { Comprometimento } \\
\text { afetivo }\end{array}$ & $\begin{array}{c}\text { Comprometimento } \\
\text { normativo }\end{array}$ & Consentimento \\
\hline Treinamento e desenvolvimento & $0,255^{* *}$ & $0,223^{* *}$ & 0,102 \\
Remuneração justa & $0,141^{* *}$ & $0,120^{* *}$ & $0,159^{* *}$ \\
Avaliação do chefe & $0,272^{* *}$ & 0,117 & $0,214^{* *}$ \\
Coordenação e controle & 0,048 & 0,029 & $0,127^{* *}$ \\
Avaliação da equipe de trabalho & $0,127^{* *}$ & 0,060 & 0,044 \\
$\mathrm{R}$ & 0,622 & 0,411 & 0,445 \\
$\mathrm{R}^{2}$ & 0,384 & 0,165 & 0,194 \\
\hline
\end{tabular}

** $\mathrm{p}<0,001$.

Psico-USF, Bragança Paulista, v. 20, n. 1, p. 51-61, jan./abr. 2015 
sente-se em débito com a organização ao perceber que ela tem investido na sua capacitação.

Já a explicação para o efeito, ainda que modesto $(\beta=0,141)$, da remuneração justa sobre o comprometimento afetivo pode ser deduzida a partir a percepção do trabalhador sobre justiça distributiva que é apontada por Meyer e Allen (1997) como uma importante variável para a explicação do comprometimento afetivo. Como a adequação da remuneração ao tipo de trabalho desempenhado pelo funcionário é um dos elementos centrais da noção de justiça organizacional, espera-se que a variável remuneração justa seja uma preditora do comprometimento.

A validade discriminante entre as duas dimensões do comprometimento não é prejudicada pelo impacto que as variáveis $\mathrm{T} \& \mathrm{D}$ e remuneração justa provocam em ambas. Como o $\mathrm{CA}$ e o $\mathrm{CN}$ são bases de um mesmo construto, é de se prever que as mesmas variáveis expliquem, em algum grau, as duas dimensões, diferindo-se apenas no valor de contribuição para cada uma. A mesma justificativa pode ser usada para explicar o tamanho do efeito das variáveis T\&D e remuneração justa para o consentimento. Por outro lado, é importante observar que, mesmo que T\&D e remuneração justa sejam consideradas preditores do $\mathrm{CA}$ e do $\mathrm{CN}$, as suas contribuições para a explicação da variação total são bastante distintas. Além disso, o CA pressupõe uma avaliação positiva do chefia e da equipe, consistentes com sua formulação teórica, ao passo que isso não se observa em relação ao $\mathrm{CN}$.

Já com relação ao consentimento, conforme o modelo proposto por Silva (2009), ele apresenta um comportamento que o afasta tanto do CA quanto do $\mathrm{CN}$ no que diz respeito tanto aos tamanhos quanto à direção dos efeitos, constituindo mais uma evidência $\mathrm{da}$ validade discriminante entre os construtos. Nesse caso, percebe-se a importância da remuneração, da avaliação do chefe e da presença de mecanismos de coordenação e controle como variáveis explicativas do consentimento, ao passo que o T\&D não impacta no resultado, o que é compatível com um vínculo passivo do trabalhador com a organização. A porção explicada da variação do consentimento é razoável (próxima a $20 \%$ ) e distinta daquela observada em relação as demais variáveis dependentes. Ou seja, de um modo geral, as variáveis preditoras dos diversos vínculos são distintas e, mesmo quando existe algum elemento em comum entre elas, seu impacto, na variação total, é distinto. Com isso, existem elementos para afirmar que, na forma proposta, os construtos apresentam validade discriminante entre si.

\section{Considerações Finais}

Este trabalho foi baseado em um estudo extensivo do tipo survey. Ainda que seus resultados sejam significativos, ele apresenta um conjunto de limitações: a amostra utilizada não é representativa da variedade dos contextos de trabalho e, portanto, os resultados não podem ser generalizados. Este estudo tomou um conjunto limitado de antecedentes, por isso, outros trabalhos são necessários para se averiguar se o padrão observado se sustenta para outras variáveis. A validade discriminante dos construtos foi investigada em relação ao modelo tridimensional do comprometimento, há que se verificar como o consentimento e o comprometimento normativo se comportam em relação a outras variáveis consequentes.

Existe uma importante corrente de investigações sobre os vínculos que o trabalhador estabelece com a organização (Pina \& Cunha, Rego, Cunha, \& Cabral-Cardoso, 2007). Nessa linha, é que se inserem os trabalhos sobre o comprometimento organizacional que têm, no modelo tridimensional de Meyer e Allen $(1991,1997)$, sua expressão mais significativa. Entretanto, vários são os problemas de natureza conceitual e metodológica que envolvem as pesquisas nessa área. Um destaque especial tem sido dado às questões sobre a validade discriminante das bases afetivas e normativas do comprometimento.

Uma tentativa de superação desses problemas é o modelo proposto por Silva (2009), que retrata a necessidade de diferenciar um trabalhador comprometido de um trabalhador obediente (Silva \& Bastos, 2010). Os indícios de validade discriminante do consentimento encontrados neste artigo sugerem que a superposição conceitual, tão frequentemente apontada na literatura entre as bases afetivas e normativas, podem sim, ser superadas pela delimitação dos vínculos passivos com a organização em um construto distinto.

Entretanto, antes que o consentimento possa ser afirmado como um tipo de vínculo específico do trabalhador com a organização há que se investigar mais profundamente qual a exata natureza dessa ligação, já que as análises realizadas apenas contemplam uma diferenciação entre as medidas propostas, mas não a adequação da medida ao construto.

Mais importante, ainda, para que o consentimento faça sentido teórico, há que se estabelecer, de forma clara, quais os comportamentos organizacionais, desejados ou indesejados, que podem ser mais bem compreendidos e previstos a partir de sua aplicação. 
Como esse é um construto novo, espera-se que, em trabalhos futuros, essas questões sejam respondidas.

\section{Referências}

Bandeira, M. L, Marques, A. L., \& Veiga, R. T. (2000). As dimensões múltiplas do comprometimento organizacional: Um estudo na ECT/MG. RAC, 4(2), 133-157.

Bar-Hayim, A., \& Berman, G. S. (1992). The dimensions of organizational commitment. Journal of Organizational Behavior, 13, 379-387.

Becker, H.S. (1960). Notes on the concept of commitment. The American Journal of Sociology, 66(1), 32-40.

Bergman, M. (2006). The relationship between affective and normative commitment: review and research agenda. Journal of Organizational Behavior, 27, 645-663.

Bastos, A. V. (1994). Comprometimento Organizacional: a estrutura dos vinculos do trabalbador com a organização, carreira e o sindicato (Tese de Doutorado não Publicada). Instituto de Psicologia da Universidade de Brasília, Brasília, Brasil.

Bastos, A. V. B., Rodrigues, A. C. A., Moscon, D. B., Silva, E. E. C., \& Pinho, A. P. M. (2013). Comprometimento no trabalho: Fundamentos para a gestão de pessoas. Em Borges, Lívia e Mourão, L. (Orgs.), O trabalho e as organizações: Atuações a partir da psicologia (pp. 270-309). Porto Alegre: Artmed.

Bastos, A. V. B., Rodrigues, A. C. A., Macambira, M. O., \& Borges-Andrade, J. E. (2014). Vínculos dos indivíduos com a organização: Análise da produção científica brasileira 2000-2010. Psicologia: Teoria e Pesquisa, 30(2), 153-162.

Blau, G. (2001). On assessing the construct validity of two multidimensional constructs. Human Resources Management Review, 11(2), 279-298.

Carvalho, P., Alves, F. J. O., Peixoto, A. L. A., \& Bastos, A. V. B. (2011). Comprometimento afetivo, de continuação e entrincheiramento: Estabelecendo limites conceituais e empíricos. Psicologia: Teoria e Prática, 13(2), 127-141.

Cooper-Hakim, A., \& Viswesvaran, C. (2005). The construct of work commitment: Testing an integrative framework. Psychological Bulletin, 131(2), 241-259.
Costa, F. M. (2005). Múltiplos comprometimentos no trabalho: Identificando padrões e explorando antecedentes entre trabalhadores de organizações agrícolas (Dissertação de Mestrado não publicada). Instituto de Psicologia da Universidade Federal da Bahia, Salvador, Brasil. Bahia.

Gellatly, I. R., Meyer, J. P., \& Luchak, A. A. (2006). Combined effects of the three commitment components on focal and discretionary behaviors: A test of Meyer and Herscovitch's propositions. Journal of Vocational Behavior, 69, 331-345.

Imran, I., Arif, I., Cheema, S., \& Azeem, M. (2014). Relationship between job satisfaction, job performance, attitude towards work, and organizational commitment. Entrepreneurship and Innovation Management Journal, 2(2), 135-144.

Janoff-Bulman, R., \& Leggatt, H. K. (2002). Culture and social obligation: When "shoulds" are perceived as "wants". Journal of Research in Personality, 36, 260-270.

Jaros, S. J. (1997). An assessment of meyer and allen's (1991) model of organizational commitment and turnover intentions. Journal of Vocational Behavior, 51, 319-337.

Klein, H. J., Becker, T. E., \& Meyer, J. P. (Eds.). (2009). Commitment in organizations: Accumulated wisdom and new directions.

Ko, J.W., Price, J. L., \& Mueller, C. W. (1997). Assessment of meyer and allen's three-component model of organizational commitment in South Korea. Journal of Applied Psychology, 82(6), 961-973.

Mathieu, J.E., \& Zajac, D.M. (1990). A review and meta-analysis of the antecedents, correlates, and consequences or organizational commitment. Psychological Bulletin, 108, 171-194.

Medeiros, C. A. F. (2003). Comprometimento organizacional: Um estudo de suas relacõos com características organizacionais e desempenho nas empresas hoteleiras (Tese de doutorado não publicada). Instituto de Psicologia da Universidade de São Paulo, São Paulo, Brasil.

Meyer, J. P. \& Allen, N. J. (1991). A three-component conceptualization of organizational commitment. Human Resource Management Review, 1, 61-89.

Meyer, J. P. \& Allen, N. J. (1997). Commitment in the workplace- theory, research and application. Advanced Topics 
in Organizational Behavior. Thousand Oaks: SAGE Publications.

Meyer, J.P. \& Herscovitch, L. (2001). Commitment in the workplace: Towards a general model. Human Resource Management Review. 11(3), 299-396.

Meyer, J.P.; Stanley, D. J.; Herscovitch, L., \& Topolnystsky, L. (2002). Affective, continuance and normative commitment to the organization: A meta-analysis of antecedents, correlates, and consequences. Journal of Vocational Behavior, 61, 20-52.

Mowday, R. T.; Steers, R. M., \& Porter, L. W. (1979). The measurement of organizational commitment. Journal of Vocational Behavior, 65, 224-247.

Mowday, R. T., Porter, L. W., \& Steers, R. M. (1982). Employee-organization linkages: The psychology of commitment, absenteeism, and turnover. New York: Academic Press.

O’Reilly III, C. \& Chatman, J. (1986). Organizational commitment and psychological attachment: the effects of compliance, identification and internalization of prosocial behavior. Journal of Applied Psychology, 72(3), 492-499.

Pina e Cunha, M. Rego, A., Cunha, R.C., \& Cabral-Cardoso, C. (2007). Manual de comportamento organizacional e gestão. Lisboa: Rh Editora.

Rodrigues, A. C. A. (2009). Do comprometimento de continuação ao entrincheiramento organizacional: $O$ percurso de validação da escala e análise da sobreposição entre os construtos. (Dissertação de mestrado não publicada), Instituto de Psicologia da Universidade Federal da Bahia, Salvador, Brasil.

Rodrigues, A. C. A., \& Bastos, A. V. B. (2012). Entrincheiramento organizacional: construção e validação de uma escala. Psicologia Reflexão e Crítica, 25(4), 688-700.

Rodrigues, A. P. G.; Bastos, A. V. B., \& Gondim, S. M. G. (2013). Comprometimento, Entrincheiramento e regulação emocional em trabalhadores do serviço público. Revista Científica Hermes, 8, 23-46.

Scheible, A.C.F.; Bastos, A. V. B., \& Rodrigues, A. C. A. (2013). Comprometimento e entrincheiramento na carreira: Integrar ou reconstruir os construtos? Uma exploração das relações à luz do desempenho. R.Adm, 48(3), 530-543.

Silva, E. E. (2009). Consentimento organizacional: Uma proposta de medida do construto. (Dissertação de mestrado não publicada), Instituto de Psicologia da Universidade Federal da Bahia, Salvador, Brasil.

Silva, E. E., \& Bastos, A.V. (2010) A Escala de consentimento organizacional: Construção e evidências de sua validade. Revista Psicologia: Organizacõos e Trabalho. 10(1), 7-22.

Solinger, O. N., Olffen, W., \& Roe, R. A. (2008). Beyond the three-component model of organizational commitment. Journal of Applied Psychology, 93(1), 70-83.

Wright, P. M., \& Kehoe R. R. (2009). Organizational-level antecedents and consequences of commitment. In Klein, H. J., Becker, T. E., \& Meyer, J. P. (Orgs), Commitment in organizations: Accumulated wisdom and new directions (pp. 285-305). Florence, KY: Routledge/Taylor and Francis Group.

Recebido em: 28/08/2013 Primeira Reformulação em: 09/10/2014 Segunda Reformulação em: 04/12/2014 Aprovado em: 10/12/2014 
Sobre os autores:

Adriano de Lemos Alves Peixoto é administrador, psicólogo, mestre em Administração (UFBA), PhD em Psicologia pela Institute of Work Psychology - University of Sheffield- UK, bolsista de pós-doutorado do programa nacional de pós-doutorado da Capes junto ao Instituto de Psicologia da UFBA.

Antônio Virgílio Bittencourt Bastos é psicólogo formado pela Universidade Federal da Bahia (1976), mestre em Educação pela Universidade Federal da Bahia (1982) e doutor em Psicologia pela Universidade de Brasília (1994), com concentração em Psicologia Organizacional e do Trabalho, e professor titular de Psicologia Social das Organizações no departamento de Psicologia da Universidade Federal da Bahia.

Icléia Santos Dorea Soares é estudante de Psicologia e bolsista de iniciação científica da Universidade Federal da Bahia.

Júlia Rodrigues Lobo é estudante de Psicologia e bolsista de iniciação científica da Universidade Federal da Bahia.

Contato com os autores:

Adriano de Lemos Alves Peixoto

Av. Ademar de Barros s/n, Campus Universitário de Ondina, pavilhão 4

CEP: 40170-110

E-mail: peixoto@hotmail.co.uk 